\title{
SISTEMA DE MIGRAÇÃO DE DADOS PARA O CONTROLE DE PROCESSOS
}

\author{
F. A. MELAZZI ${ }^{1}$, A. R. SILVA ${ }^{1}$ e N. I. BOJORGE RAMIREZ1* \\ ${ }^{1}$ Universidade Federal Fluminense, Departamento de Engenharia Química e de Petróleo \\ Rua Passo da Pátria 156, São Domingos, Niterói - RJ \\ *Email para contato: nbojorge@id.uff.br
}

\begin{abstract}
RESUMO - O objetivo deste trabalho é apresentar o desenvolvimento de um processo de implantação de máscara de entrada para os dados, a fím de possibilitar de forma automatizada, a migração de dados, oriundos do sistema supervisório da planta didática. Foi desenvolvida uma ferramenta de software, para acessar fontes de dados e fazer as transformações, validações gráficas das variáveis de processos, tratamento de erros e inserções no banco de dados. A ferramenta, a partir dos dados disponibilizados pelo sistema supervisório ou fontes de dados, e dentro de uma estratégia de controle previamente especificada dos processos na planta PD3, tem o objetivo de demonstrar didaticamente a operação das diversas malhas de controle utilizando os mesmos equipamentos e ferramentas de configuração (em software) aplicados no âmbito industrial.
\end{abstract}

Palavras-chaves: migração de dados, conversão de dados, controle de processos, Fieldbus, VBA

\section{INTRODUÇÃO}

O controle automático de processos é uma das disciplinas que tem se desenvolvido a uma velocidade vertiginosa, dando as bases ao que hoje alguns autores denominam a segunda revolução industrial (Ogata K., 2011; Smith e Corripio, 2008). O uso intensivo das técnicas de controle automático de processos tem como origem a evolução e desenvolvimento das tecnologias de medição e controle aplicadas ao ambiente industrial.

Seu estudo e aplicação tem contribuído ao reconhecimento universal de suas vantagens e benefícios associados ao âmbito industrial, que é onde se têm uma de suas maiores aplicações devido à necessidade de controlar um grande número de variáveis, somado isto à crescente complexidade dos sistemas (Vasile-Mircea et al., 2003; Moscoso-Vasquez et al. 2014), O controle automático de processos se usa fundamentalmente porque reduz o custo associado à geração de bens e serviços, incrementa a qualidade e volumes de produção de uma planta industrial entre outros benefícios associados com sua aplicação. A eliminação de erros e um aumento na segurança dos processos é outra contribuição do uso e aplicação desta técnica de controle. Neste ponto é importante destacar que anterior à aplicação massiva das técnicas de controle automático na indústria, era o homem o que aplicava suas capacidades de cálculo e sua força física para a execução do controle de um processo ou equipamento associado à produção. 
$\mathrm{Na}$ atualidade, graças ao desenvolvimento e aplicação das técnicas modernas de controle, um grande número de tarefas e cálculos associados à manipulação das variáveis tem sido delegado aos computadores, controladores e sistemas de acionamentos especializados para atingir os requerimentos do sistema. Por essas razões, o controle de processos é parte essencial da indústria moderna.

Para garantir um melhor desempenho é necessário, primeiramente, compreender de forma objetiva os dados gerados durante o processo. Poder representar a evolução das variáveis de processos, tais como a vazão, temperatura, volume, nível, composição, $\mathrm{pH}$ ao longo do processo e em torno de seus valores desejados (setpoints), assim como representar as variações requeridas nas variáveis manipuladas, dentre outros. $\mathrm{Na}$ atualidade esse procedimento é realizado mediante sistemas supervisórios, os quais permitem que sejam monitoradas e rastreadas as informações de um processo produtivo ou de uma instalação física. Tais informações são coletadas através de dispositivos de aquisição de dados e, em seguida, manipuladas, analisadas, armazenadas e, posteriormente, apresentadas ao usuário. Estes sistemas também são conhecidos como SCADA (Supervisory Control And Data Aquisition) e uma de suas funções é a aquisição de dados - que envolve a coleta e transmissão de dados desde a planta industrial (estações remotas) até às estações centrais de monitoramento. As estações remotas leem os valores dos dispositivos a eles conectados; após a leitura desses valores, segue-se a transmissão de dados em que, quer em modo de comunicação por polling, ou em modo de comunicação por interrupção (Report by Exception), os dados são transmitidos através da rede de comunicações até à estação central. O processo é concluído com o armazenamento da informação na base de dados (Huyck, 2013).

Neste contexto, qualquer engenheiro químico que está envolvido com o projeto ou na operação de plantas químicas deve ter conhecimentos mínimos de controle automático de processos. Nas grades curriculares dos cursos de Engenharia Química das distintas Universidades de Química e Escolas de Engenharias contém esta disciplina. O departamento de Engenharia Química e de Petróleo da Universidade Federal Fluminense, adquiriu e implementou uma planta didática Fielbus (PD3 - Planta Didática 3, SMAR). Essa planta é equipada com uma rede industrial Foundation Fieldbus que contém servidor OPC disponível. Essa rede conta com alguns sensores e atuadores micro processados ligados em um barramento, onde é possível aplicar várias técnicas de controle e possibilitar a simulação de um ambiente industrial próximo do real para fins de teste e validações do sistema (Bojorge Ramirez, N.I. , 2014).

O foco central deste trabalho é automatizar a etapa de tratamento de dados gerados, a fim de apresenta-los de maneira objetiva e facilmente visualizáveis para poderem ser usados no processo de uma melhor automação considerando-se as perturbações externas recorrentes.

\section{METODOLOGIA}

Utilizando-se da Planta PD3 conforme se observa na Figura 1, configurou-se um processo de aquecimento da água de um dos tanques. Especificou-se um valor de temperatura no setpoint, e este foi alterado em diferentes momentos para aumentar a complexidade dos dados obtidos. 
Figura 1 - Planta PD3 instalada no Laboratório BIOTEC - UFF

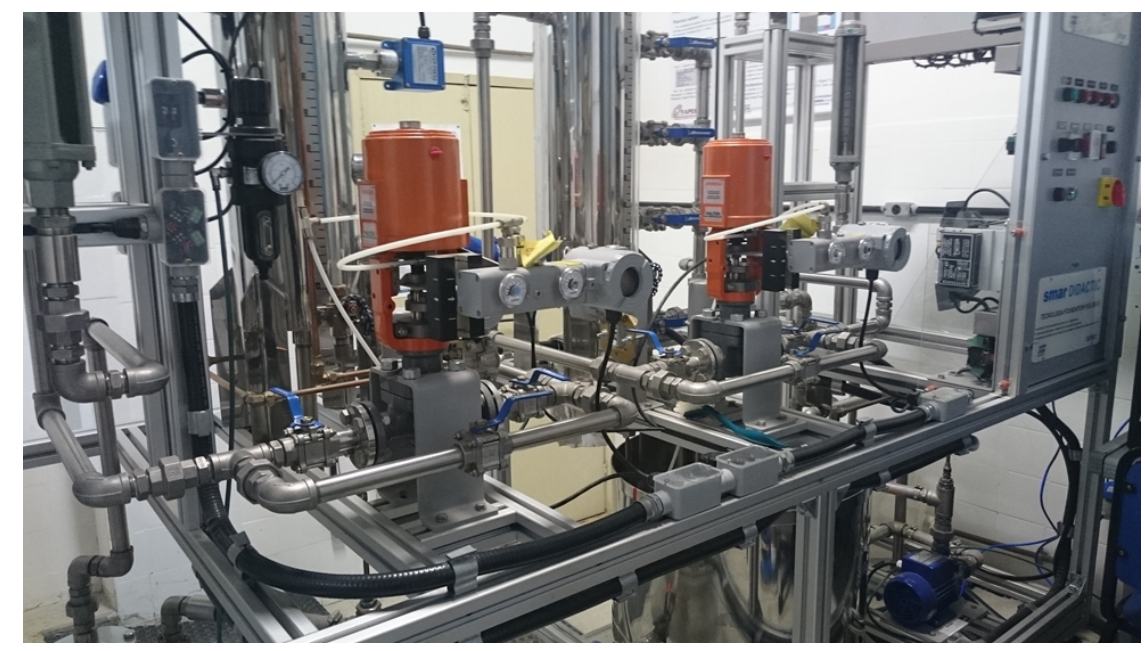

Em comunicação Fieldbus com a PD3 há um computador desktop configurado com o software System 302. Pela interface do programa é possível controlar suas automações, monitorar e extrair os dados desejados durante o processo. A extração pode ser configurada para salvar as informações que são desejadas e em diferentes intervalos de tempo. Registrouse o Setpoint, a Variável Manipulada e Variável de Processo, nesse caso a temperatura da água. $\mathrm{O}$ intervalo de tempo para extração dos dados foi de 1 minuto; um intervalo menor não daria um aumento significativo de relevância.

Terminada a obtenção dos dados, estes ficam disponibilizados em formato de arquivo .CSV (Comma-Separated Values; Valores Separados por Vírgulas), conforme se apresenta na Figura 2. Este arquivo pode ser visualizado por qualquer editor simples de texto, mas é idealmente lido por planilhas eletrônicas avançadas como Microsoft Excel, LibreOffice e semelhantes.

Figura 2 - O Registro Gravado no modo Persistent

\begin{tabular}{|c|c|}
\hline TIC31002 (DADOS CRU) - Notepad & $-\square \mid x$ \\
\hline File Edit Format View Help & \\
\hline 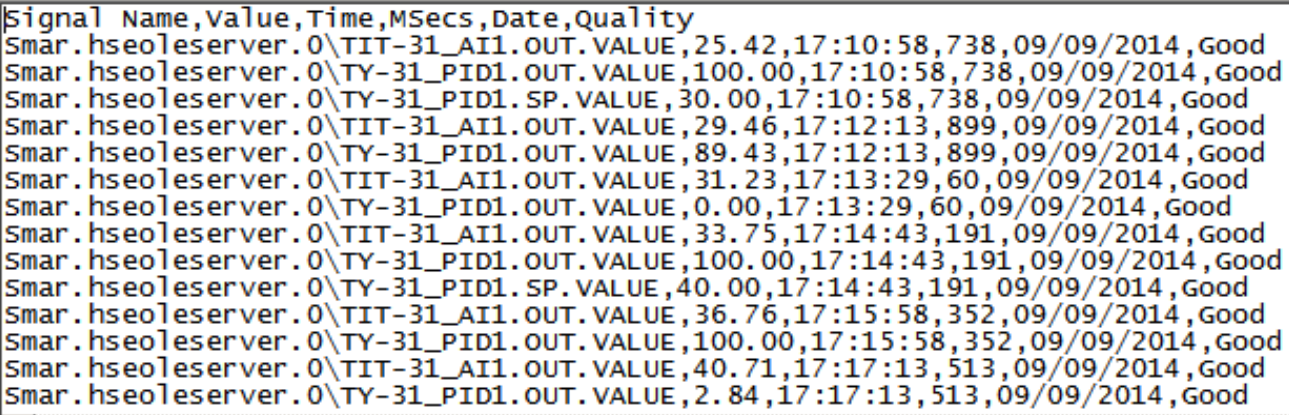 & $\Delta$ \\
\hline 4 & \\
\hline
\end{tabular}

O processo seguinte foi de tratamento e separação dos dados e criação de macros. Na automação das macros foi-se necessário programação em linguagem VBA (Visual Basic for 


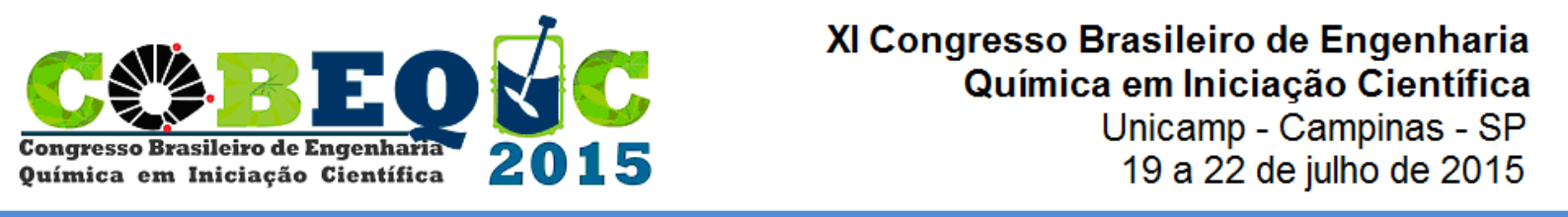

Applications) para realizar determinadas tarefas repetitivas. Uma máscara foi planejada e criada para que só seja necessário inserir os dados originais do arquivo gerado para a máscara mediante o pressionamento de um botão realizar uma sequencia de eventos para aplicar a Macro programada.

Primeiramente instruiu-se a macro para dividir em colunas os tipos de dados do arquivo gerado com as informações compiladas pelo sistema - sendo estes dados separados por vírgulas. Em seguida, separou-se cada tipo de linha de informação pela sua variável em planilhas próprias, sendo estas de Variável Manipulada, Variável de Processo ou Setpoint. Depois, focou-se em tratar cada uma das variáveis para conversão de valores e equações, definição de momento inicial e tempo decorrido de cada dado, dentre outras operações necessárias. Por fim refletiu-se as informações das colunas da planilha separadas de cada variável para uma planilha geral contendo resumidamente os dados relevantes; e o gráfico pode ser configurado a partir desta.

Para a construção do gráfico composto com múltiplas variáveis, duplicou-se os dados tabelados de Variável Manipulada e de Setpoint para que estes estivessem com a mesma quantidade de amostras de dados que a de Variável de Processo. Como só se grava os dados de uma variável quando esta se modifica, foi-se necessário repetir seus últimos valores registrados nos momentos em que estes se mantiveram constantes e não registrados.

Após a criação inicial da macro, esta foi exaustivamente testada com diferentes tipos de parâmetros. Dentre eles, diferentes períodos de tempo, quantidades de dados, Variáveis Manipuladas e Setpoints. Tudo isso para garantir que a macro vai agir como pré-determinada nas mais variadas situações.

\section{Resultados e Discussão}

O resultado final foi uma máscara automatizada programada em VBA para tratar os dados instantaneamente após o apertar de um botão. Tornando a organização dos múltiplos dados gerados pela planta de processos praticamente um sistema automático e facilitando a interpreção dos dados gerados pelos processos da planta, como se poder observar na Figura 3. 
Figura 3 - Apresentação de dados reais da planta processada mediante a máscara.

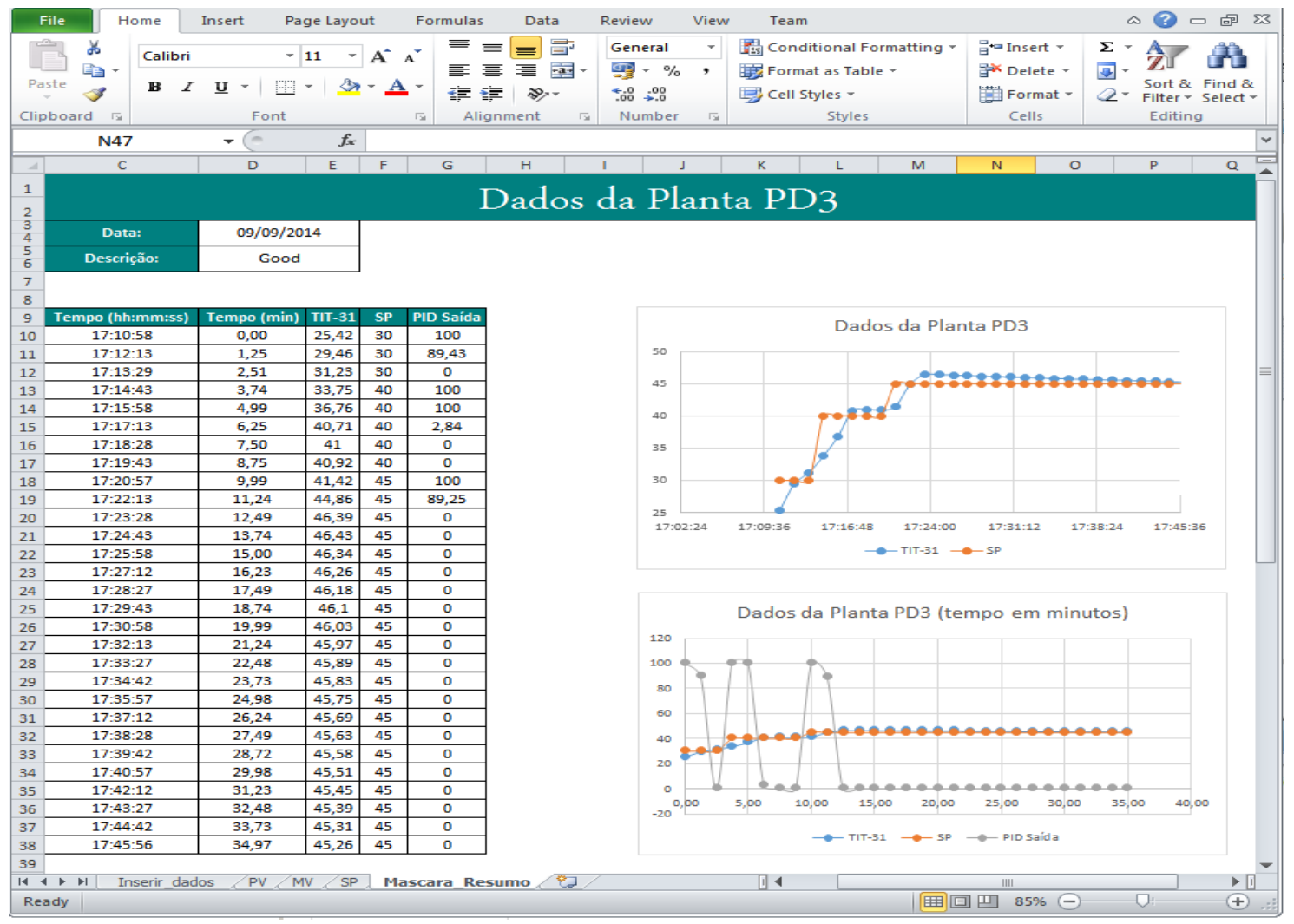

\section{Conclusão}

O tratamento automático de dados através de uma máscara pré-programada alcançou os resultados desejados. A automação para a formatação repetitiva dos dados longos agiu como o planejado tornando clara a visualização dos dados de processo. Isso facilita diversas implementações e operações para o aprendizado do sistema de controle de processo com a planta didática. Dentre eles simular teoricamente todas as variáveis da planta considerando-se realisticamente todos os distúrbios externos que possam ocorrer na realidade prática e garantir um aprendizado para os discentes do curso de Engenharia Química de casos de estudos o mais próximo com a indústria em suas pesquisas.

\section{Referências}

B.R. BAKSHI, G. STEPHANOPOULOS (1994), Representation of process trends-IV. Induction of real-time patterns from operating data for diagnosis and supervisory control, Computers \& Chemical Engineering, 18 (4), 303-332, ISSN 0098-1354, http://dx.doi.org/10.1016/0098-1354 (94)85029-1. 
BART HUYCK (2013) Model Predictive Control in the Chemical Process Industry hosted by Industries Controllers, Doctoral thesis, KU Leuven - Faculty of Engineering Science.Belgium Em: ftp://ftp.esat.kuleuven.be/pub/SISTA/ida/reports/13-161.pdf. Consultado em 27 Março 2015

BOJORGE RAMIREZ, N.I. (2014), Sistema de Monitoramento e Controle de Processos para fins Didáticos e de Pesquisas, Relatório Técnico e Cientifico, Processo $\mathrm{N}^{\mathrm{o}}$ E26/110.861/2011.

C VASILE-MIRCEA, L DAN, A. SERBAN PAUL (2003), Educational in Chemical process control using the supervisory laboratory application, Hungria Journal of industrial chemistry Veszprem, 31, 31-35.

CARLOS A. SMITH, ARMANDO B. CORRIPIO (2008), Princípios E Prática do Controle Automático de Processo - Rio de Janeiro: LTC.

HILDA M. MOSCOSO-VASQUEZ, GLORIA M. MONSALVE -BRAVO e HERNAN Alvarez (2014), Model-Based Supervisory Control Structure for Plantwide Control of a Reactor-Separator-Recycle Plant, Ind. Eng. Chem. Res., 2014, 53 (52), pp $20177-$ 20190 .DOI: 10.1021/ie502625u

K. OGATA (2011), Engenharia de Controle Moderno, 5ta Ed., LTC,

Manual do Microsoft Office Excel (2007), https://www.microsoft.com/brasil/2007office/programs/excel/guide.mspx) consultado em Agosto 2014.

SU WHAN SUNG, JIETAE LEE, IN-BEUM LEE, Process Identification and PID Control, SBN: 978-0-470-82410-8 